\title{
Existence and uniqueness of solutions for multi-term nonlinear fractional integro-differential equations
}

\author{
Dumitru Baleanu ${ }^{1,2,3^{*}}$, Sayyedeh Zahra Nazemi ${ }^{4}$ and Shahram Rezapour ${ }^{4}$
}

\author{
"Correspondence: \\ dumitru@cankaya.edu.tr \\ 'Department of Chemical and \\ Materials Engineering, Faculty of \\ Engineering, King Abdulaziz \\ University, P.O. Box 80204, Jeddah, \\ 21589, Saudi Arabia \\ ${ }^{2}$ Department of Mathematics, \\ Cankaya University, Ogretmenler \\ Cad. 14, Balgat, Ankara, 06530, \\ Turkey \\ Full list of author information is \\ available at the end of the article
}

\begin{abstract}
In this manuscript, by using the fixed point theorems, the existence and the uniqueness of solutions for multi-term nonlinear fractional integro-differential equations are reported. Two examples are presented to illustrate our results.
\end{abstract}

Keywords: Caputo fractional derivative; fixed point theorem; multi-term nonlinear fractional differential equation

\section{Introduction}

The study of fractional differential equations ranges from the theoretical aspects of existence and uniqueness of solutions to the analytic and numerical methods for finding solutions. Fractional differential equations appear naturally in a number of fields such as physics, polymer rheology, regular variational in thermodynamics, biophysics, blood flow phenomena, aerodynamics, electro-dynamics of complex medium, viscoelasticity, Bode's analysis of feedback amplifiers, capacitor theory, electrical circuits, electron-analytical chemistry, biology, control theory, fitting of experimental data, etc. An excellent account in the study of fractional differential equations can be found in [1,2] and [3]. For more details and examples, one can study [4-13] and [14]. It is considerable that there are many works about fractional integro-differential equations (see, for example, [15-18] and [19]).

In 2007, Xinwei and Landong reviewed the existence of solutions for the nonlinear fractional differential equation

$$
{ }^{c} D^{\alpha} u(t)=f\left(t, u(t),{ }^{c} D^{\beta} u(t)\right) \quad(0<t<1)
$$

with boundary values $u(0)=u^{\prime}(1)=0$ or $u^{\prime}(0)=u(1)=0$ or $u(0)=u(1)=0$, where $1<\alpha \leq$ $2,0<\beta \leq 1$, and $f$ is continuous on $[0,1] \times \mathbb{R} \times \mathbb{R}[20]$. In 2009, Su and Zhang studied the existence and uniqueness of solutions for the following nonlinear two-point fractional boundary value problem

$$
{ }^{c} D^{\alpha} u(t)=f\left(t, u(t),{ }^{c} D^{\beta} u(t)\right) \quad(0<t<1)
$$

with boundary values $a_{1} u(0)-a_{2} u^{\prime}(0)=A$ and $b_{1} u(1)+b_{2} u^{\prime}(1)=B$, where $\alpha, \beta, a_{i}, b_{i}$ $(i=1,2)$ satisfy certain conditions [21]. In 2010, Ahmad and Sivasundaram studied the 
existence of solutions for the nonlinear fractional integro-differential equation

$$
{ }^{c} D^{q} u(t)=f(t, u(t),(\phi u)(t),(\psi u)(t)) \quad(0<t<1 \text { and } 1<q \leq 2)
$$

with boundary values $u^{\prime}(0)+a u\left(\eta_{1}\right)=0, b u^{\prime}(1)+u\left(\eta_{2}\right)=0$ and $0<\eta_{1} \leq \eta_{2}<1$, where ${ }^{c} D^{q}$ is the Caputo fractional derivative, $a, b \in(0,1), f:[0,1] \times X \times X \times X \rightarrow X$ is continuous and for the mappings $\gamma, \lambda:[0,1] \times[0,1] \rightarrow[0, \infty)$ with the property $\sup _{t \in[0,1]}\left|\int_{0}^{t} \lambda(t, s) d s\right|<\infty$ and $\sup _{t \in[0,1]}\left|\int_{0}^{t} \gamma(t, s) d s\right|<\infty$, the maps $\phi$ and $\psi$ are defined by $(\phi u)(t)=\int_{0}^{t} \gamma(t, s) u(s) d s$ and $(\psi u)(t)=\int_{0}^{t} \lambda(t, s) u(s) d s$. Here, $X$ is a Banach space (see [22]).

\section{Main results}

\subsection{The basic problem}

In this paper, we study the existence and uniqueness of solutions for the multi-term nonlinear fractional integro-differential equation

$$
{ }^{c} D^{\alpha} u(t)=f\left(t, u(t),(\phi u)(t),(\psi u)(t),{ }^{c} D^{\beta_{1}} u(t),{ }^{c} D^{\beta_{2}} u(t), \ldots,{ }^{c} D^{\beta_{n}} u(t)\right) \quad(0<t<1)
$$

with boundary values $u(0)+a u(1)=0$ and $u^{\prime}(0)+b u^{\prime}(1)=0$, where $1<\alpha<2,0<\beta_{i}<1$, $\alpha-\beta_{i} \geq 1, a, b \neq-1, f:[0,1] \times \mathbb{R}^{n+3} \rightarrow \mathbb{R}$ is continuous, and for the mappings

$$
\gamma, \lambda:[0,1] \times[0,1] \rightarrow[0, \infty)
$$

with the property $\sup _{t \in[0,1]}\left|\int_{0}^{t} \lambda(t, s) d s\right|<\infty$ and $\sup _{t \in[0,1]}\left|\int_{0}^{t} \gamma(t, s) d s\right|<\infty$, the maps $\phi$ and $\psi$ are defined by $(\phi u)(t)=\int_{0}^{t} \gamma(t, s) u(s) d s$ and $(\psi u)(t)=\int_{0}^{t} \lambda(t, s) u(s) d s$. In this way, we need the following result, which has been proved in [2].

Lemma 2.1 Let $\alpha>0$ and $n=[\alpha]+1$. Then

$$
I^{\alpha c} D^{\alpha} u(t)=u(t)+c_{0}+c_{1} t+c_{2} t+\cdots+c_{n-1} t^{n-1},
$$

where $c_{0}, c_{1}, \ldots, c_{n-1}$ are some real numbers.

The proof of the following result by using Lemma 2.1 is straightforward.

Lemma 2.2 Let $y \in C[0,1], a, b \neq-1$ and $1<\alpha<2$. Then the problem ${ }^{c} D^{\alpha} u(t)=y(t)$ with boundary values $u(0)+a u(1)=0$ and $u^{\prime}(0)+b u^{\prime}(1)=0$ has the unique solution

$$
\begin{aligned}
u(t)= & \frac{1}{\Gamma(\alpha)} \int_{0}^{t}(t-s)^{\alpha-1} y(s) d s-\frac{a}{(1+a) \Gamma(\alpha)} \int_{0}^{1}(1-s)^{\alpha-1} y(s) d s \\
& +\frac{a b-b(1+a) t}{(1+a)(1+b) \Gamma(\alpha-1)} \int_{0}^{1}(1-s)^{\alpha-2} y(s) d s .
\end{aligned}
$$

\subsection{Some results on solving the problem}

Let $C(I)$ be the space of all continuous real-valued functions on $I=[0,1]$ and

$$
X=\left\{u: u \in C(I) \text { and }{ }^{c} D^{\beta_{i}} u \in C(I)\left(0<\beta_{i}<1\right) \text { for } i=1,2, \ldots, n\right\}
$$

endowed with the norm $\|u\|=\max _{t \in I}|u(t)|+\left.\sum_{i=1}^{n} \max _{t \in I}\right|^{c} D^{\beta_{i}} u(t) \mid$. It is known that $(X$, $\|\cdot\|)$ is a Banach space. 
Theorem 2.3 Assume that there exist $\kappa \in(0, \alpha-1)$ and $\mu(t) \in L^{\frac{1}{\kappa}}([0,1],(0, \infty))$ such that

$$
\begin{aligned}
& \left|f\left(t, x, y, w, u_{1}, u_{2}, \ldots, u_{n}\right)-f\left(t, x^{\prime}, y^{\prime}, w^{\prime}, v_{1}, v_{2}, \ldots, v_{n}\right)\right| \\
& \quad \leq \mu(t)\left(\left|x-x^{\prime}\right|+\left|y-y^{\prime}\right|+\left|w-w^{\prime}\right|+\left|u_{1}-v_{1}\right|+\left|u_{2}-v_{2}\right|+\cdots+\left|u_{n}-v_{n}\right|\right)
\end{aligned}
$$

for all $t \in[0,1]$ and $x, y, w, x^{\prime}, y^{\prime}, w^{\prime}, u_{1}, u_{2}, \ldots, u_{n}, v_{1}, v_{2}, \ldots, v_{n} \in \mathbb{R}$. Then problem (1) has a unique solution whenever

$$
\begin{aligned}
\Delta= & \left(1+\gamma_{0}+\lambda_{0}\right)\left[\frac{(1+2|a|) \mu^{*}}{|1+a| \Gamma(\alpha)}\left(\frac{1-\kappa}{\alpha-\kappa}\right)^{1-\kappa}+\frac{|b|(1+2|a|) \mu^{*}}{|1+a||1+b| \Gamma(\alpha-1)}\left(\frac{1-\kappa}{\alpha-\kappa-1}\right)^{1-\kappa}\right. \\
& +\sum_{i=1}^{n}\left(\frac{\Gamma(\alpha-\kappa) \mu^{*}}{\Gamma(\alpha-1) \Gamma\left(\alpha-\beta_{i}-\kappa+1\right)}\left(\frac{1-\kappa}{\alpha-\kappa-1}\right)^{1-\kappa}\right. \\
& \left.\left.+\frac{|b| \mu^{*}}{|1+b| \Gamma\left(2-\beta_{i}\right) \Gamma(\alpha-1)}\left(\frac{1-\kappa}{\alpha-\kappa-1}\right)^{1-\kappa}\right)\right]<1,
\end{aligned}
$$

where $\gamma_{0}=\sup _{t \in I}\left|\int_{0}^{t} \gamma(t, s) d s\right|, \lambda_{0}=\sup _{t \in I}\left|\int_{0}^{t} \lambda(t, s) d s\right|, \mu^{*}=\left(\int_{0}^{1}(\mu(s))^{\frac{1}{\kappa}} d s\right)^{\kappa}$.

Proof Define the mapping $F: X \rightarrow X$ by

$$
\begin{aligned}
(F u)(t)= & \int_{0}^{t} \frac{(t-s)^{\alpha-1}}{\Gamma(\alpha)} f\left(s, u(s),(\phi u)(s),(\psi u)(s),{ }^{c} D^{\beta_{1}} u(s),{ }^{c} D^{\beta_{2}} u(s), \ldots,{ }^{c} D^{\beta_{n}} u(s)\right) d s \\
& -\frac{a}{(1+a)} \int_{0}^{1} \frac{(1-s)^{\alpha-1}}{\Gamma(\alpha)} \\
& \times f\left(s, u(s),(\phi u)(s),(\psi u)(s),{ }^{c} D^{\beta_{1}} u(s),{ }^{c} D^{\beta_{2}} u(s), \ldots,{ }^{c} D^{\beta_{n}} u(s)\right) d s \\
& +\frac{a b-b(1+a) t}{(1+a)(1+b)} \int_{0}^{1} \frac{(1-s)^{\alpha-2}}{\Gamma(\alpha-1)} \\
& \times f\left(s, u(s),(\phi u)(s),(\psi u)(s),{ }^{c} D^{\beta_{1}} u(s),{ }^{c} D^{\beta_{2}} u(s), \ldots,{ }^{c} D^{\beta_{n}} u(s)\right) d s .
\end{aligned}
$$

For each $u, v \in X$ and $t \in[0,1]$, by using the Hölder inequality, we have

$$
\begin{aligned}
\mid(F u)( & t)-(F v)(t) \mid \\
= & \mid \int_{0}^{t} \frac{(t-s)^{\alpha-1}}{\Gamma(\alpha)}\left(f\left(s, u(s),(\phi u)(s),(\psi u)(s),{ }^{c} D^{\beta_{1}} u(s),{ }^{c} D^{\beta_{2}} u(s), \ldots,{ }^{c} D^{\beta_{n}} u(s)\right)\right. \\
& \left.-f\left(s, v(s),(\phi v)(s),(\psi v)(s),{ }^{c} D^{\beta_{1}} v(s),{ }^{c} D^{\beta_{2}} v(s), \ldots,{ }^{c} D^{\beta_{n}} v(s)\right)\right) d s \\
& \quad-\frac{a}{(1+a)} \int_{0}^{1} \frac{(1-s)^{\alpha-1}}{\Gamma(\alpha)}\left(f\left(s, u(s),(\phi u)(s),(\psi u)(s),{ }^{c} D^{\beta_{1}} u(s),{ }^{c} D^{\beta_{2}} u(s), \ldots,{ }^{c} D^{\beta_{n}} u(s)\right)\right. \\
& \left.-f\left(s, v(s),(\phi v)(s),(\psi v)(s),{ }^{c} D^{\beta_{1}} v(s),{ }^{c} D^{\beta_{2}} v(s), \ldots,{ }^{c} D^{\beta_{n}} v(s)\right)\right) d s \\
& +\frac{a b-b(1+a) t}{(1+a)(1+b)} \int_{0}^{1} \frac{(1-s){ }^{\alpha-2}}{\Gamma(\alpha-1)}\left(f \left(s, u(s),(\phi u)(s),(\psi u)(s),{ }^{c} D^{\beta_{1}} u(s),{ }^{c} D^{\beta_{2}} u(s)\right.\right. \\
& \left.\left.\quad \ldots,{ }^{c} D^{\beta_{n}} u(s)\right)-f\left(s, v(s),(\phi v)(s),(\psi v)(s),{ }^{c} D^{\beta_{1}} v(s),{ }^{c} D^{\beta_{2}} v(s), \ldots,{ }^{c} D^{\beta_{n}} v(s)\right)\right) d s \mid \\
\leq & \int_{0}^{t} \frac{(t-s)^{\alpha-1}}{\Gamma(\alpha)} \mid f\left(s, u(s),(\phi u)(s),(\psi u)(s),{ }^{c} D^{\beta_{1}} u(s),{ }^{c} D^{\beta_{2}} u(s), \ldots,{ }^{c} D^{\beta_{n}} u(s)\right)
\end{aligned}
$$


$-f\left(s, v(s),(\phi v)(s),(\psi v)(s),{ }^{c} D^{\beta_{1}} v(s),{ }^{c} D^{\beta_{2}} v(s), \ldots,{ }^{c} D^{\beta_{n}} v(s)\right) \mid d s$

$+\frac{|a|}{|1+a|} \int_{0}^{1} \frac{(1-s)^{\alpha-1}}{\Gamma(\alpha)} \mid f\left(s, u(s),(\phi u)(s),(\psi u)(s),{ }^{c} D^{\beta_{1}} u(s),{ }^{c} D^{\beta_{2}} u(s), \ldots,{ }^{c} D^{\beta_{n}} u(s)\right)$

$-f\left(s, v(s),(\phi v)(s),(\psi v)(s),{ }^{c} D^{\beta_{1}} v(s),{ }^{c} D^{\beta_{2}} v(s), \ldots,{ }^{c} D^{\beta_{n}} v(s)\right) \mid d s$

$+\frac{|a b-b(1+a) t|}{|1+a||1+b|} \int_{0}^{1} \frac{(1-s)^{\alpha-2}}{\Gamma(\alpha-1)} \mid f\left(s, u(s),(\phi u)(s),(\psi u)(s),{ }^{c} D^{\beta_{1}} u(s),{ }^{c} D^{\beta_{2}} u(s)\right.$,

$\left.\ldots,{ }^{c} D^{\beta_{n}} u(s)\right)-f\left(s, v(s),(\phi v)(s),(\psi v)(s),{ }^{c} D^{\beta_{1}} v(s),{ }^{c} D^{\beta_{2}} v(s), \ldots,{ }^{c} D^{\beta_{n}} v(s)\right) \mid d s$

$\leq \int_{0}^{t} \frac{(t-s)^{\alpha-1}}{\Gamma(\alpha)} \mu(s)(|u(s)-v(s)|+|(\phi u)(s)-(\phi v)(s)|+|(\psi u)(s)-(\psi v)(s)|$

$\left.+\left|{ }^{c} D^{\beta_{1}} u(s)-{ }^{c} D^{\beta_{1}} v(s)\right|+\left|{ }^{c} D^{\beta_{2}} u(s)-{ }^{c} D^{\beta_{2}} v(s)\right|+\cdots+\left|{ }^{c} D^{\beta_{n}} u(s)-{ }^{c} D^{\beta_{n}} v(s)\right|\right) d s$

$+\frac{|a|}{|1+a|} \int_{0}^{1} \frac{(1-s)^{\alpha-1}}{\Gamma(\alpha)} \mu(s)(|u(s)-v(s)|+|(\phi u)(s)-(\phi v)(s)|+|(\psi u)(s)-(\psi v)(s)|$

$\left.+\left|{ }^{c} D^{\beta_{1}} u(s)-{ }^{c} D^{\beta_{1}} v(s)\right|+\left|{ }^{c} D^{\beta_{2}} u(s)-{ }^{c} D^{\beta_{2}} v(s)\right|+\cdots+\left|{ }^{c} D^{\beta_{n}} u(s)-{ }^{c} D^{\beta_{n}} v(s)\right|\right) d s$

$+\frac{|b|(1+2|a|)}{|1+a||1+b|} \int_{0}^{1} \frac{(1-s)^{\alpha-2}}{\Gamma(\alpha-1)} \mu(s)(|u(s)-v(s)|+|(\phi u)(s)-(\phi v)(s)|$

$+|(\psi u)(s)-(\psi v)(s)|+\left|{ }^{c} D^{\beta_{1}} u(s)-{ }^{c} D^{\beta_{1}} v(s)\right|$

$\left.+\left|{ }^{c} D^{\beta_{2}} u(s)-{ }^{c} D^{\beta_{2}} v(s)\right|+\cdots+\left|{ }^{c} D^{\beta_{n}} u(s)-{ }^{c} D^{\beta_{n}} v(s)\right|\right) d s$

$\leq \frac{\left(1+\gamma_{0}+\lambda_{0}\right)\|u-v\|}{\Gamma(\alpha)} \int_{0}^{t}(t-s)^{\alpha-1} \mu(s) d s$

$+\frac{|a|\left(1+\gamma_{0}+\lambda_{0}\right)\|u-v\|}{|1+a| \Gamma(\alpha)} \int_{0}^{1}(1-s)^{\alpha-1} \mu(s) d s$

$+\frac{|b|(1+2|a|)\left(1+\gamma_{0}+\lambda_{0}\right)\|u-v\|}{|1+a||1+b| \Gamma(\alpha-1)} \int_{0}^{1}(1-s)^{\alpha-2} \mu(s) d s$

$\leq \frac{\left(1+\gamma_{0}+\lambda_{0}\right)\|u-v\|}{\Gamma(\alpha)}\left(\int_{0}^{t}\left((t-s)^{\alpha-1}\right)^{\frac{1}{1-\kappa}} d s\right)^{1-\kappa}\left(\int_{0}^{t}(\mu(s))^{\frac{1}{\kappa}} d s\right)^{\kappa}$

$+\frac{|a|\left(1+\gamma_{0}+\lambda_{0}\right)\|u-v\|}{|1+a| \Gamma(\alpha)}\left(\int_{0}^{1}\left((1-s)^{\alpha-1}\right)^{\frac{1}{1-\kappa}} d s\right)^{1-\kappa}\left(\int_{0}^{1}(\mu(s))^{\frac{1}{\kappa}} d s\right)^{\kappa}$

$+\frac{|b|(1+2|a|)\left(1+\gamma_{0}+\lambda_{0}\right)\|u-v\|}{|1+a||1+b| \Gamma(\alpha-1)}$

$\times\left(\int_{0}^{1}\left((1-s)^{\alpha-2}\right)^{\frac{1}{1-\kappa}} d s\right)^{1-\kappa}\left(\int_{0}^{1}(\mu(s))^{\frac{1}{\kappa}} d s\right)^{\kappa}$

$\leq \frac{\mu^{*}\left(1+\gamma_{0}+\lambda_{0}\right)\|u-v\|}{\Gamma(\alpha)}\left(\frac{1-\kappa}{\alpha-\kappa}\right)^{1-\kappa}+\frac{|a| \mu^{*}\left(1+\gamma_{0}+\lambda_{0}\right)\|u-v\|}{|1+a| \Gamma(\alpha)}\left(\frac{1-\kappa}{\alpha-\kappa}\right)^{1-\kappa}$

$+\frac{|b|(1+2|a|) \mu^{*}\left(1+\gamma_{0}+\lambda_{0}\right)\|u-v\|}{|1+a||1+b| \Gamma(\alpha-1)}\left(\frac{1-\kappa}{\alpha-\kappa-1}\right)^{1-\kappa}$

$\leq\left(1+\gamma_{0}+\lambda_{0}\right)\left[\frac{(1+2|a|) \mu^{*}}{|1+a| \Gamma(\alpha)}\left(\frac{1-\kappa}{\alpha-\kappa}\right)^{1-\kappa}\right.$

$\left.+\frac{|b|(1+2|a|) \mu^{*}}{|1+a||1+b| \Gamma(\alpha-1)}\left(\frac{1-\kappa}{\alpha-\kappa-1}\right)^{1-\kappa}\right]\|u-v\|$. 
Also, we have

$$
\begin{aligned}
& \left|{ }^{c} D^{\beta_{i}}(F u)(t)-{ }^{c} D^{\beta_{i}}(F v)(t)\right| \\
& =\left|\int_{0}^{t} \frac{(t-s)^{-\beta_{i}}}{\Gamma\left(1-\beta_{i}\right)}(F u)^{\prime}(s) d s-\int_{0}^{t} \frac{(t-s)^{-\beta_{i}}}{\Gamma\left(1-\beta_{i}\right)}(F v)^{\prime}(s) d s\right| \\
& =\mid \int_{0}^{t} \frac{(t-s)^{-\beta_{i}}}{\Gamma\left(1-\beta_{i}\right)}\left(\int_{0}^{s} \frac{(s-\tau)^{\alpha-2}}{\Gamma(\alpha-1)}\right. \\
& \times f\left(\tau, u(\tau),(\phi u)(\tau),(\psi u)(\tau),{ }^{c} D^{\beta_{1}} u(\tau),{ }^{c} D^{\beta_{2}} u(\tau), \ldots,{ }^{c} D^{\beta_{n}} u(\tau)\right) d \tau \\
& -\frac{b}{1+b} \int_{0}^{1} \frac{(1-\tau)^{\alpha-2}}{\Gamma(\alpha-1)} \\
& \left.\times f\left(\tau, u(\tau),(\phi u)(\tau),(\psi u)(\tau),{ }^{c} D^{\beta_{1}} u(\tau),{ }^{c} D^{\beta_{2}} u(\tau), \ldots,{ }^{c} D^{\beta_{n}} u(\tau)\right) d \tau\right) d s \\
& -\int_{0}^{t} \frac{(t-s)^{-\beta_{i}}}{\Gamma\left(1-\beta_{i}\right)}\left(\int_{0}^{s} \frac{(s-\tau)^{\alpha-2}}{\Gamma(\alpha-1)}\right. \\
& \times f\left(\tau, v(\tau),(\phi v)(\tau),(\psi v)(\tau),{ }^{c} D^{\beta_{1}} v(\tau),{ }^{c} D^{\beta_{2}} v(\tau), \ldots,{ }^{c} D^{\beta_{n}} v(\tau)\right) d \tau \\
& -\frac{b}{1+b} \int_{0}^{1} \frac{(1-\tau)^{\alpha-2}}{\Gamma(\alpha-1)} \\
& \left.\times f\left(\tau, v(\tau),(\phi v)(\tau),(\psi v)(\tau),{ }^{c} D^{\beta_{1}} v(\tau),{ }^{c} D^{\beta_{2}} v(\tau), \ldots,{ }^{c} D^{\beta_{n}} v(\tau)\right) d \tau\right) d s \mid \\
& \leq \int_{0}^{t} \frac{(t-s)^{-\beta_{i}}}{\Gamma\left(1-\beta_{i}\right)}\left(\int_{0}^{s} \frac{(s-\tau)^{\alpha-2}}{\Gamma(\alpha-1)}\right. \\
& \times \mid f\left(\tau, u(\tau),(\phi u)(\tau),(\psi u)(\tau),{ }^{c} D^{\beta_{1}} u(\tau),{ }^{c} D^{\beta_{2}} u(\tau), \ldots,{ }^{c} D^{\beta_{n}} u(\tau)\right) \\
& \left.-f\left(\tau, v(\tau),(\phi v)(\tau),(\psi v)(\tau),{ }^{c} D^{\beta_{1}} v(\tau),{ }^{c} D^{\beta_{2}} v(\tau), \ldots,{ }^{c} D^{\beta_{n}} v(\tau)\right) \mid d \tau\right) d s \\
& +\frac{|b|}{|1+b|} \int_{0}^{t} \frac{(t-s)^{-\beta_{i}}}{\Gamma\left(1-\beta_{i}\right)} \\
& \times\left(\int_{0}^{1} \frac{(1-\tau)^{\alpha-2}}{\Gamma(\alpha-1)} \mid f\left(\tau, u(\tau),(\phi u)(\tau),(\psi u)(\tau),{ }^{c} D^{\beta_{1}} u(\tau),{ }^{c} D^{\beta_{2}} u(\tau), \ldots,{ }^{c} D^{\beta_{n}} u(\tau)\right)\right. \\
& \left.-f\left(\tau, v(\tau),(\phi v)(\tau),(\psi v)(\tau),{ }^{c} D^{\beta_{1}} v(\tau),{ }^{c} D^{\beta_{2}} v(\tau), \ldots,{ }^{c} D^{\beta_{n}} v(\tau)\right) \mid d \tau\right) d s \\
& \leq \frac{\left(1+\gamma_{0}+\lambda_{0}\right)\|u-v\|}{\Gamma\left(1-\beta_{i}\right) \Gamma(\alpha-1)} \int_{0}^{t}(t-s)^{-\beta_{i}}\left(\int_{0}^{s}(s-\tau)^{\alpha-2} \mu(\tau) d \tau\right) d s \\
& +\frac{|b|\left(1+\gamma_{0}+\lambda_{0}\right)\|u-v\|}{|1+b| \Gamma\left(1-\beta_{i}\right) \Gamma(\alpha-1)} \int_{0}^{t}(t-s)^{-\beta_{i}}\left(\int_{0}^{1}(1-\tau)^{\alpha-2} \mu(\tau) d \tau\right) d s \\
& \leq \frac{\mu^{*}\left(1+\gamma_{0}+\lambda_{0}\right)\|u-v\|}{\Gamma\left(1-\beta_{i}\right) \Gamma(\alpha-1)}\left(\frac{1-\kappa}{\alpha-\kappa-1}\right)^{1-\kappa} \int_{0}^{t}(t-s)^{-\beta_{i}} s^{\alpha-\kappa-1} d s \\
& +\frac{|b| \mu^{*}\left(1+\gamma_{0}+\lambda_{0}\right)\|u-v\|}{|1+b| \Gamma\left(1-\beta_{i}\right) \Gamma(\alpha-1)}\left(\frac{1-\kappa}{\alpha-\kappa-1}\right)^{1-\kappa} \int_{0}^{t}(t-s)^{-\beta_{i}} d s \\
& \leq \frac{\mu^{*}\left(1+\gamma_{0}+\lambda_{0}\right)\|u-v\|}{\Gamma\left(1-\beta_{i}\right) \Gamma(\alpha-1)}\left(\frac{1-\kappa}{\alpha-\kappa-1}\right)^{1-\kappa} \int_{0}^{1}(1-\xi)^{-\beta_{i}} \xi^{\alpha-\kappa-1} d \xi \\
& +\frac{|b| \mu^{*}\left(1+\gamma_{0}+\lambda_{0}\right)\|u-v\|}{|1+b| \Gamma\left(2-\beta_{i}\right) \Gamma(\alpha-1)}\left(\frac{1-\kappa}{\alpha-\kappa-1}\right)^{1-\kappa} \text {. }
\end{aligned}
$$


Since $B\left(\alpha-\kappa, 1-\beta_{i}\right)=\int_{0}^{1}(1-\xi)^{-\beta_{i}} \xi^{\alpha-\kappa-1} d \xi=\frac{\Gamma(\alpha-\kappa) \Gamma\left(1-\beta_{i}\right)}{\Gamma\left(\alpha-\beta_{i}-\kappa+1\right)}$, we obtain

$$
\begin{aligned}
\left|{ }^{c} D^{\beta_{i}}(F u)(t)-{ }^{c} D^{\beta_{i}}(F v)(t)\right| \leq & \left(1+\gamma_{0}+\lambda_{0}\right)\left[\frac{\Gamma(\alpha-\kappa) \mu^{*}}{\Gamma(\alpha-1) \Gamma\left(\alpha-\beta_{i}-\kappa+1\right)}\left(\frac{1-\kappa}{\alpha-\kappa-1}\right)^{1-\kappa}\right. \\
& \left.+\frac{|b| \mu^{*}}{|1+b| \Gamma\left(2-\beta_{i}\right) \Gamma(\alpha-1)}\left(\frac{1-\kappa}{\alpha-\kappa-1}\right)^{1-\kappa}\right]\|u-v\|
\end{aligned}
$$

for all $i=1,2, \ldots, n$. Hence, we get

$$
\begin{aligned}
\| F u- & F v \| \\
\leq & \left(1+\gamma_{0}+\lambda_{0}\right)\left[\frac{(1+2|a|) \mu^{*}}{|1+a| \Gamma(\alpha)}\left(\frac{1-\kappa}{\alpha-\kappa}\right)^{1-\kappa}+\frac{|b|(1+2|a|) \mu^{*}}{|1+a||1+b| \Gamma(\alpha-1)}\left(\frac{1-\kappa}{\alpha-\kappa-1}\right)^{1-\kappa}\right. \\
& +\sum_{i=1}^{n}\left(\frac{\Gamma(\alpha-\kappa) \mu^{*}}{\Gamma(\alpha-1) \Gamma\left(\alpha-\beta_{i}-\kappa+1\right)}\left(\frac{1-\kappa}{\alpha-\kappa-1}\right)^{1-\kappa}\right. \\
& \left.\left.+\frac{|b| \mu^{*}}{|1+b| \Gamma\left(2-\beta_{i}\right) \Gamma(\alpha-1)}\left(\frac{1-\kappa}{\alpha-\kappa-1}\right)^{1-\kappa}\right)\right]\|u-v\|=\Delta\|u-v\| .
\end{aligned}
$$

Since $\Delta<1, F$ is a contraction mapping, therefore, by using the Banach contraction principle, $F$ has a unique fixed point, which is the unique solution of problem (1) by using Lemma 2.2.

Corollary 2.4 Assume that there exists $L>0$ such that

$$
\begin{aligned}
& \left|f\left(t, x, y, w, u_{1}, u_{2}, \ldots, u_{n}\right)-f\left(t, x^{\prime}, y^{\prime}, w^{\prime}, v_{1}, v_{2}, \ldots, v_{n}\right)\right| \\
& \quad \leq L\left(\left|x-x^{\prime}\right|+\left|y-y^{\prime}\right|+\left|w-w^{\prime}\right|+\left|u_{1}-v_{1}\right|+\left|u_{2}-v_{2}\right|+\cdots+\left|u_{n}-v_{n}\right|\right)
\end{aligned}
$$

for all $t \in[0,1]$ and $x, y, w, x^{\prime}, y^{\prime}, w^{\prime}, u_{1}, u_{2}, \ldots, u_{n}, v_{1}, v_{2}, \ldots, v_{n} \in \mathbb{R}$. Then problem (1) has a unique solution whenever

$$
\begin{aligned}
\left(1+\gamma_{0}+\lambda_{0}\right)\left[\frac{(1+2|a|)(1+(\alpha+1)|b|) L}{|1+a||1+b| \Gamma(\alpha+1)}\right. \\
\left.\quad+\sum_{i=1}^{n}\left(\frac{L}{\Gamma\left(\alpha-\beta_{i}+1\right)}+\frac{|b| L}{|1+b| \Gamma\left(2-\beta_{i}\right) \Gamma(\alpha)}\right)\right]<1,
\end{aligned}
$$

where $\gamma_{0}=\sup _{t \in I}\left|\int_{0}^{t} \gamma(t, s) d s\right|, \lambda_{0}=\sup _{t \in I}\left|\int_{0}^{t} \lambda(t, s) d s\right|$.

Now, we restate the Schauder's fixed point theorem, which is needed to prove next result (see Theorem 1.10.16 in [23]).

Theorem 2.5 Let E be a closed, convex and bounded subset of a Banach space $X$, and let $F: E \rightarrow E$ be a continuous mapping such that $F(E)$ is a relatively compact subset of $X$. Then $F$ has a fixed point in $E$. 
Theorem 2.6 Let $f:[0,1] \times \mathbb{R}^{n+3} \rightarrow \mathbb{R}$ be a continuous function such that there exists a constant $l \in(0, \alpha-1)$ and a real-valued function $m(t) \in L^{\frac{1}{l}}([0,1],(0, \infty))$ such that

$$
\begin{aligned}
& \left|f\left(t, x, y, w, u_{1}, u_{2}, \ldots, u_{n}\right)\right| \\
& \quad \leq m(t)+d|x|^{\rho}+d^{\prime}|y|^{\rho^{\prime}}+d^{\prime \prime}|w|^{\rho^{\prime \prime}}+d_{1}\left|u_{1}\right|^{\rho_{1}}+d_{2}\left|u_{2}\right|^{\rho_{2}}+\cdots+d_{n}\left|u_{n}\right|^{\rho_{n}},
\end{aligned}
$$

where $d, d^{\prime}, d^{\prime \prime}, d_{i} \geq 0$ and $0<\rho, \rho^{\prime}, \rho^{\prime \prime}, \rho_{i}<1$ for $i=1,2, \ldots, n$, or

$$
\begin{aligned}
& \left|f\left(t, x, y, w, u_{1}, u_{2}, \ldots, u_{n}\right)\right| \\
& \quad \leq d|x|^{\rho}+d^{\prime}|y|^{\rho^{\prime}}+d^{\prime \prime}|w|^{\rho^{\prime \prime}}+d_{1}\left|u_{1}\right|^{\rho_{1}}+d_{2}\left|u_{2}\right|^{\rho_{2}}+\cdots+d_{n}\left|u_{n}\right|^{\rho_{n}},
\end{aligned}
$$

where $d, d^{\prime}, d^{\prime \prime}, d_{i}>0$ and $\rho, \rho^{\prime}, \rho^{\prime \prime}, \rho_{i}>1$ for $i=1,2, \ldots, n$. Then problem (1) has a solution.

Proof First, suppose that $f$ satisfy condition $(*)$. Define $B_{r}=\{u \in X,\|u\| \leq r\}$, where

$$
\begin{aligned}
r \geq & \max \left\{((n+4) A d)^{\frac{1}{1-\rho}},\left((n+4) A d^{\prime} \gamma_{0}^{p^{\prime}}\right)^{\frac{1}{1-\rho^{\prime}}},\left((n+4) A d^{\prime \prime} \lambda_{0}^{p^{\prime \prime}}\right)^{\frac{1}{1-\rho^{\prime \prime}}},\left((n+4) A d_{1}\right)^{\frac{1}{1-\rho_{1}}},\right. \\
& \left.\left((n+4) A d_{2}\right)^{\frac{1}{1-\rho_{2}}}, \ldots,\left((n+4) A d_{n}\right)^{\frac{1}{1-\rho_{n}}},(n+4) K\right\} \\
K= & \frac{(1+2|a|) M}{|1+a| \Gamma(\alpha)}\left(\frac{1-l}{\alpha-l}\right)^{1-l}+\frac{|b|(1+2|a|) M}{|1+a||1+b| \Gamma(\alpha-1)}\left(\frac{1-l}{\alpha-l-1}\right)^{1-l} \\
& +\sum_{i=1}^{n}\left(\frac{\Gamma(\alpha-l) M}{\Gamma(\alpha-1) \Gamma\left(\alpha-\beta_{i}-l+1\right)}\left(\frac{1-l}{\alpha-l-1}\right)^{1-l}\right. \\
& \left.+\frac{|b| M}{|1+b| \Gamma\left(2-\beta_{i}\right) \Gamma(\alpha-1)}\left(\frac{1-l}{\alpha-l-1}\right)^{1-l}\right), \\
A= & \frac{(1+2|a|)(1+(1+\alpha)|b|)}{|1+a||1+b| \Gamma(\alpha+1)}+\sum_{i=1}^{n}\left(\frac{1}{\Gamma\left(\alpha-\beta_{i}+1\right)}+\frac{|b|}{|1+b| \Gamma(\alpha) \Gamma\left(2-\beta_{i}\right)}\right)
\end{aligned}
$$

and $M=\left(\int_{0}^{1}(m(t))^{\frac{1}{l}} d s\right)^{l}$. Note that $B_{r}$ is a closed, bounded and convex subset of the Banach space $X$. For each $u \in B_{r}$, we have

$$
\begin{aligned}
|(F u)(t)|= & \mid \int_{0}^{t} \frac{(t-s)^{\alpha-1}}{\Gamma(\alpha)} f\left(s, u(s),(\phi u)(s),(\psi u)(s),{ }^{c} D^{\beta_{1}} u(s),{ }^{c} D^{\beta_{2}} u(s), \ldots,{ }^{c} D^{\beta_{n}} u(s)\right) d s \\
& -\frac{a}{(1+a)} \int_{0}^{1} \frac{(1-s)^{\alpha-1}}{\Gamma(\alpha)} \\
& \times f\left(s, u(s),(\phi u)(s),(\psi u)(s),{ }^{c} D^{\beta_{1}} u(s),{ }^{c} D^{\beta_{2}} u(s), \ldots,{ }^{c} D^{\beta_{n}} u(s)\right) d s \\
& +\frac{a b-b(1+a) t}{(1+a)(1+b)} \int_{0}^{1} \frac{(1-s)^{\alpha-2}}{\Gamma(\alpha-1)} \\
& \times f\left(s, u(s),(\phi u)(s),(\psi u)(s),{ }^{c} D^{\beta_{1}} u(s),{ }^{c} D^{\beta_{2}} u(s), \ldots,{ }^{c} D^{\beta_{n}} u(s)\right) d s \mid \\
\leq & \int_{0}^{t} \frac{(t-s)^{\alpha-1}}{\Gamma(\alpha)}\left|f\left(s, u(s),(\phi u)(s),(\psi u)(s),{ }^{c} D^{\beta_{1}} u(s),{ }^{c} D^{\beta_{2}} u(s), \ldots,{ }^{c} D^{\beta_{n}} u(s)\right)\right| d s \\
& +\frac{|a|}{|1+a|} \int_{0}^{1} \frac{(1-s)^{\alpha-1}}{\Gamma(\alpha)}
\end{aligned}
$$


Baleanu et al. Advances in Difference Equations 2013, 2013:368

Page 8 of 17

http://www.advancesindifferenceequations.com/content/2013/1/368

$$
\begin{aligned}
& \times\left|f\left(s, u(s),(\phi u)(s),(\psi u)(s),{ }^{c} D^{\beta_{1}} u(s),{ }^{c} D^{\beta_{2}} u(s), \ldots,{ }^{c} D^{\beta_{n}} u(s)\right)\right| d s \\
& +\frac{|b|(1+2|a|)}{|1+a||1+b|} \int_{0}^{1} \frac{(1-s)^{\alpha-2}}{\Gamma(\alpha-1)} \\
& \times\left|f\left(s, u(s),(\phi u)(s),(\psi u)(s),{ }^{c} D^{\beta_{1}} u(s),{ }^{c} D^{\beta_{2}} u(s), \ldots,{ }^{c} D^{\beta_{n}} u(s)\right)\right| d s \\
& \leq \int_{0}^{t} \frac{(t-s)^{\alpha-1}}{\Gamma(\alpha)} m(s) d s \\
& +\left(d r^{\rho}+d^{\prime} \gamma_{0}^{p^{\prime}} r^{\rho^{\prime}}+d^{\prime \prime} \lambda_{0}^{p^{\prime \prime}} r^{\rho^{\prime \prime}}+d_{1} r^{\rho_{1}}+d_{2} r^{\rho_{2}}+\cdots+d_{n} r^{\rho_{n}}\right) \int_{0}^{t} \frac{(t-s)^{\alpha-1}}{\Gamma(\alpha)} d s \\
& +\frac{|a|}{|1+a|} \int_{0}^{1} \frac{(1-s)^{\alpha-1}}{\Gamma(\alpha)} m(s) d s \\
& +\frac{|a|}{|1+a|}\left(d r^{\rho}+d^{\prime} \gamma_{0}^{p^{\prime}} r^{\rho^{\prime}}+d^{\prime \prime} \lambda_{0}^{p^{\prime \prime}} r^{\rho^{\prime \prime}}+d_{1} r^{\rho_{1}}+d_{2} r^{\rho_{2}}+\cdots+d_{n} r^{\rho_{n}}\right) \\
& \times \int_{0}^{1} \frac{(1-s)^{\alpha-1}}{\Gamma(\alpha)} d s+\frac{|b|(1+2|a|)}{|1+a||1+b|} \int_{0}^{1} \frac{(1-s)^{\alpha-2}}{\Gamma(\alpha-1)} m(s) d s \\
& +\frac{|b|(1+2|a|)}{|1+a||1+b|}\left(d r^{\rho}+d^{\prime} \gamma_{0}^{p^{\prime}} r^{\rho^{\prime}}+d^{\prime \prime} \lambda_{0}^{p^{\prime \prime}} r^{\rho^{\prime \prime}}+d_{1} r^{\rho_{1}}+d_{2} r^{\rho_{2}}+\cdots+d_{n} r^{\rho_{n}}\right) \\
& \times \int_{0}^{1} \frac{(1-s)^{\alpha-2}}{\Gamma(\alpha-1)} d s \\
& \leq \frac{1}{\Gamma(\alpha)}\left(\int_{0}^{t}\left((t-s)^{\alpha-1}\right)^{\frac{1}{1-l}} d s\right)^{1-l}\left(\int_{0}^{t}(m(s))^{\frac{1}{l}} d s\right)^{l} \\
& +\frac{|a|}{|1+a| \Gamma(\alpha)}\left(\int_{0}^{1}\left((1-s)^{\alpha-1}\right)^{\frac{1}{1-l}} d s\right)^{1-l}\left(\int_{0}^{1}(m(s))^{\frac{1}{l}} d s\right)^{l} \\
& +\frac{|b|(1+2|a|)}{|1+a||1+b| \Gamma(\alpha-1)}\left(\int_{0}^{1}\left((1-s)^{\alpha-2}\right)^{\frac{1}{1-l}} d s\right)^{1-l}\left(\int_{0}^{1}(m(s))^{\frac{1}{l}} d s\right)^{l} \\
& +\frac{(1+2|a|)(1+(1+\alpha)|b|)}{|1+a||1+b| \Gamma(\alpha+1)} \\
& \times\left(d r^{\rho}+d^{\prime} \gamma_{0}^{p^{\prime}} r^{\rho^{\prime}}+d^{\prime \prime} \lambda_{0}^{p^{\prime \prime}} r^{\rho^{\prime \prime}}+d_{1} r^{\rho_{1}}+d_{2} r^{\rho_{2}}+\cdots+d_{n} r^{\rho_{n}}\right) \\
& \leq \frac{(1+2|a|) M}{|1+a| \Gamma(\alpha)}\left(\frac{1-l}{\alpha-l}\right)^{1-l}+\frac{|b|(1+2|a|) M}{|1+a||1+b| \Gamma(\alpha-1)}\left(\frac{1-l}{\alpha-l-1}\right)^{1-l} \\
& +\frac{(1+2|a|)(1+(1+\alpha)|b|)}{|1+a||1+b| \Gamma(\alpha+1)} \\
& \times\left(d r^{\rho}+d^{\prime} \gamma_{0}^{p^{\prime}} r^{\rho^{\prime}}+d^{\prime \prime} \lambda_{0}^{p^{\prime \prime}} r^{\rho^{\prime \prime}}+d_{1} r^{\rho_{1}}+d_{2} r^{\rho_{2}}+\cdots+d_{n} r^{\rho_{n}}\right) .
\end{aligned}
$$

Also, we have

$$
\begin{aligned}
\left|{ }^{c} D^{\beta_{i}}(F u)(t)\right|= & \left|\int_{0}^{t} \frac{(t-s)^{-\beta_{i}}}{\Gamma\left(1-\beta_{i}\right)}(F u)^{\prime}(s) d s\right| \\
= & \mid \int_{0}^{t} \frac{(t-s)^{-\beta_{i}}}{\Gamma\left(1-\beta_{i}\right)}\left(\int_{0}^{s} \frac{(s-\tau)^{\alpha-2}}{\Gamma(\alpha-1)}\right. \\
& \times f\left(\tau, u(\tau),(\phi u)(\tau),(\psi u)(\tau),{ }^{c} D^{\beta_{1}} u(\tau),{ }^{c} D^{\beta_{2}} u(\tau), \ldots,{ }^{c} D^{\beta_{n}} u(\tau)\right) d \tau \\
& -\frac{b}{1+b} \int_{0}^{1} \frac{(1-\tau)^{\alpha-2}}{\Gamma(\alpha-1)}
\end{aligned}
$$


Baleanu et al. Advances in Difference Equations 2013, 2013:368

Page 9 of 17

http://www.advancesindifferenceequations.com/content/2013/1/368

$$
\begin{aligned}
& \times f(\tau, u(\tau),(\phi u)(\tau),(\psi u)(\tau), \\
& \left.\left.{ }^{c} D^{\beta_{1}} u(\tau),{ }^{c} D^{\beta_{2}} u(\tau), \ldots,{ }^{c} D^{\beta_{n}} u(\tau)\right) d \tau\right) d s \mid \\
& \leq \int_{0}^{t} \frac{(t-s)^{-\beta_{i}}}{\Gamma\left(1-\beta_{i}\right)}\left(\int_{0}^{s} \frac{(s-\tau)^{\alpha-2}}{\Gamma(\alpha-1)} \mid f(\tau, u(\tau),(\phi u)(\tau),(\psi u)(\tau),\right. \\
& \left.\left.{ }^{c} D^{\beta_{1}} u(\tau),{ }^{c} D^{\beta_{2}} u(\tau), \ldots,{ }^{c} D^{\beta_{n}} u(\tau)\right) \mid d \tau\right) d s \\
& +\frac{|b|}{|1+b|} \int_{0}^{t} \frac{(t-s)^{-\beta_{i}}}{\Gamma\left(1-\beta_{i}\right)}\left(\int_{0}^{1} \frac{(1-\tau)^{\alpha-2}}{\Gamma(\alpha-1)} \mid f(\tau, u(\tau),(\phi u)(\tau),(\psi u)(\tau),\right. \\
& \left.\left.{ }^{c} D^{\beta_{1}} u(\tau),{ }^{c} D^{\beta_{2}} u(\tau), \ldots,{ }^{c} D^{\beta_{n}} u(\tau)\right) \mid d \tau\right) d s \\
& \leq \int_{0}^{t} \frac{(t-s)^{-\beta_{i}}}{\Gamma\left(1-\beta_{i}\right)}\left(\int_{0}^{s} \frac{(s-\tau)^{\alpha-2}}{\Gamma(\alpha-1)} m(\tau) d \tau\right) d s \\
& +\left(d r^{\rho}+d^{\prime} \gamma_{0}^{p^{\prime}} r^{\rho^{\prime}}+d^{\prime \prime} \lambda_{0}^{p^{\prime \prime}} r^{\rho^{\prime \prime}}+d_{1} r^{\rho_{1}}+d_{2} r^{\rho_{2}}+\cdots+d_{n} r^{\rho_{n}}\right) \\
& \times \int_{0}^{t} \frac{(t-s)^{-\beta_{i}}}{\Gamma\left(1-\beta_{i}\right)}\left(\int_{0}^{s} \frac{(s-\tau)^{\alpha-2}}{\Gamma(\alpha-1)} d \tau\right) d s \\
& +\frac{|b|}{|1+b|} \int_{0}^{t} \frac{(t-s)^{-\beta_{i}}}{\Gamma\left(1-\beta_{i}\right)}\left(\int_{0}^{1} \frac{(1-\tau)^{\alpha-2}}{\Gamma(\alpha-1)} m(\tau) d \tau\right) d s \\
& +\frac{|b|}{|1+b|}\left(d r^{\rho}+d^{\prime} \gamma_{0}^{p^{\prime}} r^{\rho^{\prime}}+d^{\prime \prime} \lambda_{0}^{p^{\prime \prime}} r^{\rho^{\prime \prime}}+d_{1} r^{\rho_{1}}+d_{2} r^{\rho_{2}}+\cdots+d_{n} r^{\rho_{n}}\right) \\
& \times \int_{0}^{t} \frac{(t-s)^{-\beta_{i}}}{\Gamma\left(1-\beta_{i}\right)}\left(\int_{0}^{1} \frac{(1-\tau)^{\alpha-2}}{\Gamma(\alpha-1)} d \tau\right) d s \\
& \leq \frac{1}{\Gamma(\alpha-1) \Gamma\left(1-\beta_{i}\right)} \int_{0}^{t}(t-s)^{-\beta_{i}} \\
& \times\left[\left(\int_{0}^{s}\left((s-\tau)^{\alpha-2}\right)^{\frac{1}{1-l}} d \tau\right)^{1-l}\left(\int_{0}^{s}(m(\tau))^{\frac{1}{l}} d \tau\right)^{l}\right] d s \\
& +\frac{\left(d r^{\rho}+d^{\prime} \gamma_{0}^{p^{\prime}} r^{\rho^{\prime}}+d^{\prime \prime} \lambda_{0}^{p^{\prime \prime}} r^{\rho^{\prime \prime}}+d_{1} r^{\rho_{1}}+d_{2} r^{\rho_{2}}+\cdots+d_{n} r^{\rho_{n}}\right)}{\Gamma(\alpha) \Gamma\left(1-\beta_{i}\right)} \\
& \times \int_{0}^{t}(t-s)^{-\beta_{i}} s^{\alpha-1} d s+\frac{|b|}{|1+b| \Gamma(\alpha-1) \Gamma\left(1-\beta_{i}\right)} \\
& \times \int_{0}^{t}(t-s)^{-\beta_{i}}\left[\left(\int_{0}^{1}\left((1-\tau)^{\alpha-2}\right)^{\frac{1}{1-l}} d \tau\right)^{1-l}\left(\int_{0}^{1}(m(\tau))^{\frac{1}{l}} d \tau\right)^{l}\right] d s \\
& +\frac{|b|\left(d r^{\rho}+d^{\prime} \gamma_{0}^{p^{\prime}} r^{\rho^{\prime}}+d^{\prime \prime} \lambda_{0}^{p^{\prime \prime}} r^{\rho^{\prime \prime}}+d_{1} r^{\rho_{1}}+d_{2} r^{\rho_{2}}+\cdots+d_{n} r^{\rho_{n}}\right)}{|1+b| \Gamma(\alpha) \Gamma\left(2-\beta_{i}\right)} \\
& \leq \frac{M}{\Gamma(\alpha-1) \Gamma\left(1-\beta_{i}\right)}\left(\frac{1-l}{\alpha-l-1}\right)^{1-l} \int_{0}^{t}(t-s)^{-\beta_{i}} s^{\alpha-l-1} d s \\
& +\frac{\left(d r^{\rho}+d^{\prime} \gamma_{0}^{p^{\prime}} r^{\rho^{\prime}}+d^{\prime \prime} \lambda_{0}^{p^{\prime \prime}} r^{\rho^{\prime \prime}}+d_{1} r^{\rho_{1}}+d_{2} r^{\rho_{2}}+\cdots+d_{n} r^{\rho_{n}}\right)}{\Gamma(\alpha) \Gamma\left(1-\beta_{i}\right)} \\
& \times \int_{0}^{t}(t-s)^{-\beta_{i}} s^{\alpha-1} d s
\end{aligned}
$$


Baleanu et al. Advances in Difference Equations 2013, 2013:368

Page 10 of 17

http://www.advancesindifferenceequations.com/content/2013/1/368

$$
\begin{aligned}
& +\frac{|b| M}{|1+b| \Gamma(\alpha-1) \Gamma\left(1-\beta_{i}\right)}\left(\frac{1-l}{\alpha-l-1}\right)^{1-l} \int_{0}^{t}(t-s)^{-\beta_{i}} d s \\
& +\frac{|b|\left(d r^{\rho}+d^{\prime} \gamma_{0}^{p^{\prime}} r^{\rho^{\prime}}+d^{\prime \prime} \lambda_{0}^{p^{\prime \prime}} r^{\rho^{\prime \prime}}+d_{1} r^{\rho_{1}}+d_{2} r^{\rho_{2}}+\cdots+d_{n} r^{\rho_{n}}\right)}{|1+b| \Gamma(\alpha) \Gamma\left(2-\beta_{i}\right)} \\
& \leq \frac{M}{\Gamma(\alpha-1) \Gamma\left(1-\beta_{i}\right)}\left(\frac{1-l}{\alpha-l-1}\right)^{1-l} \int_{0}^{1}(1-\xi)^{-\beta_{i}} \xi^{\alpha-l-1} d \xi \\
& +\frac{\left(d r^{\rho}+d^{\prime} \gamma_{0}^{p^{\prime}} r^{\rho^{\prime}}+d^{\prime \prime} \lambda_{0}^{p^{\prime \prime}} r^{\rho^{\prime \prime}}+d_{1} r^{\rho_{1}}+d_{2} r^{\rho_{2}}+\cdots+d_{n} r^{\rho_{n}}\right)}{\Gamma(\alpha) \Gamma\left(1-\beta_{i}\right)} \\
& \quad \times \int_{0}^{1}(1-\xi)^{-\beta_{i}} \xi^{\alpha-1} d \xi \\
& +\frac{|b| M}{|1+b| \Gamma(\alpha-1) \Gamma\left(2-\beta_{i}\right)}\left(\frac{1-l}{\alpha-l-1}\right)^{1-l} \\
& +\frac{|b|\left(d r^{\rho}+d^{\prime} \gamma_{0}^{p^{\prime}} r^{\rho^{\prime}}+d^{\prime \prime} \lambda_{0}^{p^{\prime \prime}} r^{\rho^{\prime \prime}}+d_{1} r^{\rho_{1}}+d_{2} r^{\rho_{2}}+\cdots+d_{n} r^{\rho_{n}}\right)}{|1+b| \Gamma(\alpha) \Gamma\left(2-\beta_{i}\right)}
\end{aligned} .
$$

Since $B\left(\alpha-l, 1-\beta_{i}\right)=\int_{0}^{1}(1-\xi)^{-\beta_{i}} \xi^{\alpha-l-1} d \xi=\frac{\Gamma(\alpha-l) \Gamma\left(1-\beta_{i}\right)}{\Gamma\left(\alpha-\beta_{i}-l+1\right)}$ and, on the other hand, $B(\alpha, 1-$ $\left.\beta_{i}\right)=\int_{0}^{1}(1-\xi)^{-\beta_{i}} \xi^{\alpha-1} d \xi=\frac{\Gamma(\alpha) \Gamma\left(1-\beta_{i}\right)}{\Gamma\left(\alpha-\beta_{i}+1\right)}$, we conclude that

$$
\begin{aligned}
\left|{ }^{c} D^{\beta_{i}}(F u)(t)\right| \leq & \frac{\Gamma(\alpha-l) M}{\Gamma(\alpha-1) \Gamma\left(\alpha-\beta_{i}-l+1\right)}\left(\frac{1-l}{\alpha-l-1}\right)^{1-l} \\
& +\frac{|b| M}{|1+b| \Gamma(\alpha-1) \Gamma\left(2-\beta_{i}\right)}\left(\frac{1-l}{\alpha-l-1}\right)^{1-l} \\
& +\frac{d r^{\rho}+d^{\prime} \gamma_{0}^{p^{\prime}} r^{\rho^{\prime}}+d^{\prime \prime} \lambda_{0}^{p^{\prime \prime}} r^{\rho^{\prime \prime}}+d_{1} r^{\rho_{1}}+d_{2} r^{\rho_{2}}+\cdots+d_{n} r^{\rho_{n}}}{\Gamma\left(\alpha-\beta_{i}+1\right)} \\
& +\frac{|b|\left(d r^{\rho}+d^{\prime} \gamma_{0}^{p^{\prime}} r^{\rho^{\prime}}+d^{\prime \prime} \lambda_{0}^{p^{\prime \prime}} r^{\rho^{\prime \prime}}+d_{1} r^{\rho_{1}}+d_{2} r^{\rho_{2}}+\cdots+d_{n} r^{\rho_{n}}\right)}{|1+b| \Gamma(\alpha) \Gamma\left(2-\beta_{i}\right)}
\end{aligned}
$$

for all $i=1,2, \ldots, n$. Thus,

$$
\begin{aligned}
\|F u\| \leq & \frac{(1+2|a|) M}{|1+a| \Gamma(\alpha)}\left(\frac{1-l}{\alpha-l}\right)^{1-l}+\frac{|b|(1+2|a|) M}{|1+a||1+b| \Gamma(\alpha-1)}\left(\frac{1-l}{\alpha-l-1}\right)^{1-l} \\
& +\sum_{i=1}^{n}\left[\frac{\Gamma(\alpha-l) M}{\Gamma(\alpha-1) \Gamma\left(\alpha-\beta_{i}-l+1\right)}\left(\frac{1-l}{\alpha-l-1}\right)^{1-l}\right. \\
& \left.+\frac{|b| M}{|1+b| \Gamma(\alpha-1) \Gamma\left(2-\beta_{i}\right)}\left(\frac{1-l}{\alpha-l-1}\right)^{1-l}\right] \\
& +\left(d r^{\rho}+d^{\prime} \gamma_{0}^{p^{\prime}} r^{\rho^{\prime}}+d^{\prime \prime} \lambda_{0}^{p^{\prime \prime}} r^{\rho^{\prime \prime}}+d_{1} r^{\rho_{1}}+d_{2} r^{\rho_{2}}+\cdots+d_{n} r^{\rho_{n}}\right) \\
& \times\left(\frac{(1+2|a|)(1+(1+\alpha)|b|)}{|1+a||1+b| \Gamma(\alpha+1)}+\sum_{i=1}^{n}\left[\frac{1}{\Gamma\left(\alpha-\beta_{i}+1\right)}+\frac{11+b \mid \Gamma(\alpha) \Gamma\left(2-\beta_{i}\right)}{\mid 1+b^{\prime}}\right]\right) \\
= & K+\left(d r^{\rho}+d^{\prime} \gamma_{0}^{p^{\prime}} r^{\rho^{\prime}}+d^{\prime \prime} \lambda_{0}^{p^{\prime \prime}} r^{\rho^{\prime \prime}}+d_{1} r^{\rho_{1}}+d_{2} r^{\rho_{2}}+\cdots+d_{n} r^{\rho_{n}}\right) A \\
\leq & \frac{r}{n+4} \times(n+4)=r .
\end{aligned}
$$


Hence, $F$ maps $B_{r}$ into $B_{r}$. Now, suppose that $f$ satisfy the second condition. In this case, choose

$$
\begin{aligned}
0< & r \\
\leq & \min \left\{\left(\frac{1}{(n+3) A d}\right)^{\frac{1}{\rho-1}},\left(\frac{1}{(n+3) A d^{\prime} \gamma_{0}^{p^{\prime}}}\right)^{\frac{1}{\rho^{\prime}-1}},\left(\frac{1}{(n+3) A d^{\prime \prime} \lambda_{0}^{p^{\prime \prime}}}\right)^{\frac{1}{\rho^{\prime \prime}-1}},\right. \\
& \left.\left(\frac{1}{(n+3) A d_{1}}\right)^{\frac{1}{\rho_{1}-1}},\left(\frac{1}{(n+3) A d_{2}}\right)^{\frac{1}{\rho_{2}-1}}, \ldots,\left(\frac{1}{(n+3) A d_{n}}\right)^{\frac{1}{\rho_{n}-1}}\right\} .
\end{aligned}
$$

By using similar arguments, one can show that $\|F u\| \leq \frac{r}{n+3} \times(n+3)=r$, and so $F$ maps $B_{r}$ into $B_{r}$. Since $f$ is continuous, it is easy to get that $F$ is also continuous. Now, we show that $F$ is completely continuous operator on $B_{r}$. For each $u \in B_{r}$, put

$$
N=\max _{t \in I}\left\{f\left(t, u(t),(\phi u)(t),(\psi u)(t),{ }^{c} D^{\beta_{1}} u(t),{ }^{c} D^{\beta_{2}} u(t), \ldots,{ }^{c} D^{\beta_{n}} u(t)\right)\right\}+1 .
$$

For each $t_{1}, t_{2} \in I$ with $t_{1}<t_{2}$, we have

$$
\begin{aligned}
&\left|(F u)\left(t_{2}\right)-(F u)\left(t_{1}\right)\right| \\
&=\mid \int_{0}^{t_{2}} \frac{\left(t_{2}-s\right)^{\alpha-1}}{\Gamma(\alpha)} f\left(s, u(s),(\phi u)(s),(\psi u)(s),{ }^{c} D^{\beta_{1}} u(s),{ }^{c} D^{\beta_{2}} u(s), \ldots,{ }^{c} D^{\beta_{n}} u(s)\right) d s \\
& \quad-\int_{0}^{t_{1}} \frac{\left(t_{1}-s\right)^{\alpha-1}}{\Gamma(\alpha)} f\left(s, u(s),(\phi u)(s),(\psi u)(s),{ }^{c} D^{\beta_{1}} u(s),{ }^{c} D^{\beta_{2}} u(s), \ldots,{ }^{c} D^{\beta_{n}} u(s)\right) d s \\
& \quad+\frac{b\left(t_{1}-t_{2}\right)}{1+b} \int_{0}^{1} \frac{(1-s)^{\alpha-2}}{\Gamma(\alpha-1)} \\
& \quad \times f\left(s, u(s),(\phi u)(s),(\psi u)(s),{ }^{c} D^{\beta_{1}} u(s),{ }^{c} D^{\beta_{2}} u(s), \ldots,{ }^{c} D^{\beta_{n}} u(s)\right) d s \mid \\
&=\mid \int_{0}^{t_{1}} \frac{\left(t_{2}-s\right)^{\alpha-1}}{\Gamma(\alpha)} f\left(s, u(s),(\phi u)(s),(\psi u)(s),{ }^{c} D^{\beta_{1}} u(s),{ }^{c} D^{\beta_{2}} u(s), \ldots,{ }^{c} D^{\beta_{n}} u(s)\right) d s \\
& \quad+\int_{t_{1}}^{t_{2}} \frac{\left(t_{2}-s\right)^{\alpha-1}}{\Gamma(\alpha)} f\left(s, u(s),(\phi u)(s),(\psi u)(s),{ }^{c} D^{\beta_{1}} u(s),{ }^{c} D^{\beta_{2}} u(s), \ldots,{ }^{c} D^{\beta_{n}} u(s)\right) d s \\
& \quad-\int_{0}^{t_{1}} \frac{\left(t_{1}-s\right)^{\alpha-1}}{\Gamma(\alpha)} f\left(s, u(s),(\phi u)(s),(\psi u)(s),{ }^{c} D^{\beta_{1}} u(s),{ }^{c} D^{\beta_{2}} u(s), \ldots,{ }^{c} D^{\beta_{n}} u(s)\right) d s \\
& \quad+\frac{b\left(t_{1}-t_{2}\right)}{1+b} \int_{0}^{1} \frac{(1-s)^{\alpha-2}}{\Gamma(\alpha-1)} \\
& \quad+\frac{|b|\left(t_{2}-t_{1}\right)}{|1+b|} \int_{0}^{1} \frac{(1-s)^{\alpha-2}}{\Gamma(\alpha-1)} \\
& \quad+\int_{0}^{t_{1}} \frac{\left(t_{2}-s\right)^{\alpha-1}-\left(t_{1}-s\right)(s-1}{\Gamma(\alpha)} \\
& \quad \times\left|f\left(s, u(s),(\phi u)(s),(\psi u)(s),{ }^{c} D^{\beta_{1}} u(s),{ }^{c} D^{\beta_{2}} u(s), \ldots,{ }^{c} D^{\beta_{n}} u(s)\right)\right| d s \\
& t_{2} \frac{\left(t_{2}-s\right)^{\alpha-1}}{\Gamma(\alpha)}\left|f\left(s, u(s),(\phi u)(s),(\psi u)(s),{ }^{c} D^{\beta_{1}} u(s),{ }^{c} D^{\beta_{2}} u(s), \ldots,{ }^{c} D^{\beta_{n}} u(s)\right)\right| d s \\
&\left.\mid(\psi u)(s),{ }^{c} D^{\beta_{1}} u(s),{ }^{c} D^{\beta_{2}} u(s), \ldots,{ }^{c} D^{\beta_{n}} u(s)\right) d s \mid
\end{aligned}
$$




$$
\begin{aligned}
& \times\left|f\left(s, u(s),(\phi u)(s),(\psi u)(s),{ }^{c} D^{\beta_{1}} u(s),{ }^{c} D^{\beta_{2}} u(s), \ldots,{ }^{c} D^{\beta_{n}} u(s)\right)\right| d s \\
\leq & N \int_{0}^{t_{1}} \frac{\left(t_{2}-s\right)^{\alpha-1}-\left(t_{1}-s\right)^{\alpha-1}}{\Gamma(\alpha)} d s+N \int_{t_{1}}^{t_{2}} \frac{\left(t_{2}-s\right)^{\alpha-1}}{\Gamma(\alpha)} d s \\
& +\frac{N|b|\left(t_{2}-t_{1}\right)}{|1+b|} \int_{0}^{1} \frac{(1-s)^{\alpha-2}}{\Gamma(\alpha-1)} d s \\
= & \frac{N}{\Gamma(\alpha+1)}\left(t_{2}^{\alpha}-t_{1}^{\alpha}\right)+\frac{N|b|}{|1+b| \Gamma(\alpha)}\left(t_{2}-t_{1}\right) .
\end{aligned}
$$

On the other hand, for each $i \in\{1,2, \ldots, n\}$, we have

$$
\begin{aligned}
& \left|{ }^{c} D^{\beta_{i}}(F u)\left(t_{2}\right)-{ }^{c} D^{\beta_{i}}(F u)\left(t_{1}\right)\right| \\
& =\left|\int_{0}^{t_{2}} \frac{\left(t_{2}-s\right)^{-\beta_{i}}}{\Gamma\left(1-\beta_{i}\right)}(F u)^{\prime}(s) d s-\int_{0}^{t_{1}} \frac{\left(t_{1}-s\right)^{-\beta_{i}}}{\Gamma\left(1-\beta_{i}\right)}(F u)^{\prime}(s) d s\right| \\
& =\mid \int_{0}^{t_{2}} \frac{\left(t_{2}-s\right)^{-\beta_{i}}}{\Gamma\left(1-\beta_{i}\right)}\left(\int_{0}^{s} \frac{(s-\tau)^{\alpha-2}}{\Gamma(\alpha-1)}\right. \\
& \times f\left(\tau, u(\tau),(\phi u)(\tau),(\psi u)(\tau),{ }^{c} D^{\beta_{1}} u(\tau),{ }^{c} D^{\beta_{2}} u(\tau), \ldots,{ }^{c} D^{\beta_{n}} u(\tau)\right) d \tau \\
& -\frac{b}{1+b} \int_{0}^{1} \frac{(1-\tau)^{\alpha-2}}{\Gamma(\alpha-1)} \\
& \left.\times f\left(\tau, u(\tau),(\phi u)(\tau),(\psi u)(\tau),{ }^{c} D^{\beta_{1}} u(\tau),{ }^{c} D^{\beta_{2}} u(\tau), \ldots,{ }^{c} D^{\beta_{n}} u(\tau)\right) d \tau\right) d s \\
& -\int_{0}^{t_{1}} \frac{\left(t_{1}-s\right)^{-\beta_{i}}}{\Gamma\left(1-\beta_{i}\right)}\left(\int_{0}^{s} \frac{(s-\tau)^{\alpha-2}}{\Gamma(\alpha-1)}\right. \\
& \times f\left(\tau, u(\tau),(\phi u)(\tau),(\psi u)(\tau),{ }^{c} D^{\beta_{1}} u(\tau),{ }^{c} D^{\beta_{2}} u(\tau), \ldots,{ }^{c} D^{\beta_{n}} u(\tau)\right) d \tau \\
& -\frac{b}{1+b} \int_{0}^{1} \frac{(1-\tau)^{\alpha-2}}{\Gamma(\alpha-1)} \\
& \left.\times f\left(\tau, u(\tau),(\phi u)(\tau),(\psi u)(\tau),{ }^{c} D^{\beta_{1}} u(\tau),{ }^{c} D^{\beta_{2}} u(\tau), \ldots,{ }^{c} D^{\beta_{n}} u(\tau)\right) d \tau\right) d s \\
& =\mid \int_{0}^{t_{1}} \frac{\left(t_{2}-s\right)^{-\beta_{i}}}{\Gamma\left(1-\beta_{i}\right)}\left(\int_{0}^{s} \frac{(s-\tau)^{\alpha-2}}{\Gamma(\alpha-1)}\right. \\
& \times f\left(\tau, u(\tau),(\phi u)(\tau),(\psi u)(\tau),{ }^{c} D^{\beta_{1}} u(\tau),{ }^{c} D^{\beta_{2}} u(\tau), \ldots,{ }^{c} D^{\beta_{n}} u(\tau)\right) d \tau \\
& -\frac{b}{1+b} \int_{0}^{1} \frac{(1-\tau)^{\alpha-2}}{\Gamma(\alpha-1)} \\
& \left.\times f\left(\tau, u(\tau),(\phi u)(\tau),(\psi u)(\tau),{ }^{c} D^{\beta_{1}} u(\tau),{ }^{c} D^{\beta_{2}} u(\tau), \ldots,{ }^{c} D^{\beta_{n}} u(\tau)\right) d \tau\right) d s \\
& +\int_{t_{1}}^{t_{2}} \frac{\left(t_{2}-s\right)^{-\beta_{i}}}{\Gamma\left(1-\beta_{i}\right)}\left(\int_{0}^{s} \frac{(s-\tau)^{\alpha-2}}{\Gamma(\alpha-1)}\right. \\
& \times f\left(\tau, u(\tau),(\phi u)(\tau),(\psi u)(\tau),{ }^{c} D^{\beta_{1}} u(\tau),{ }^{c} D^{\beta_{2}} u(\tau), \ldots,{ }^{c} D^{\beta_{n}} u(\tau)\right) d \tau \\
& -\frac{b}{1+b} \int_{0}^{1} \frac{(1-\tau)^{\alpha-2}}{\Gamma(\alpha-1)} \\
& \left.\times f\left(\tau, u(\tau),(\phi u)(\tau),(\psi u)(\tau),{ }^{c} D^{\beta_{1}} u(\tau),{ }^{c} D^{\beta_{2}} u(\tau), \ldots,{ }^{c} D^{\beta_{n}} u(\tau)\right) d \tau\right) d s
\end{aligned}
$$




$$
\begin{aligned}
& -\int_{0}^{t_{1}} \frac{\left(t_{1}-s\right)^{-\beta_{i}}}{\Gamma\left(1-\beta_{i}\right)}\left(\int_{0}^{s} \frac{(s-\tau)^{\alpha-2}}{\Gamma(\alpha-1)}\right. \\
& \times f\left(\tau, u(\tau),(\phi u)(\tau),(\psi u)(\tau),{ }^{c} D^{\beta_{1}} u(\tau),{ }^{c} D^{\beta_{2}} u(\tau), \ldots,{ }^{c} D^{\beta_{n}} u(\tau)\right) d \tau \\
& -\frac{b}{1+b} \int_{0}^{1} \frac{(1-\tau)^{\alpha-2}}{\Gamma(\alpha-1)} \\
& \left.\times f\left(\tau, u(\tau),(\phi u)(\tau),(\psi u)(\tau),{ }^{c} D^{\beta_{1}} u(\tau),{ }^{c} D^{\beta_{2}} u(\tau), \ldots,{ }^{c} D^{\beta_{n}} u(\tau)\right) d \tau\right) d s \mid \\
& =\mid \int_{0}^{t_{1}} \frac{\left(t_{2}-s\right)^{-\beta_{i}}-\left(t_{1}-s\right)^{-\beta_{i}}}{\Gamma\left(1-\beta_{i}\right)} \\
& \times\left(\int_{0}^{s} \frac{(s-\tau)^{\alpha-2}}{\Gamma(\alpha-1)} f(\tau, u(\tau),(\phi u)(\tau),(\psi u)(\tau),\right. \\
& \left.{ }^{c} D^{\beta_{1}} u(\tau),{ }^{c} D^{\beta_{2}} u(\tau), \ldots,{ }^{c} D^{\beta_{n}} u(\tau)\right) d \tau \\
& -\frac{b}{1+b} \int_{0}^{1} \frac{(1-\tau)^{\alpha-2}}{\Gamma(\alpha-1)} \\
& \left.\times f\left(\tau, u(\tau),(\phi u)(\tau),(\psi u)(\tau),{ }^{c} D^{\beta_{1}} u(\tau),{ }^{c} D^{\beta_{2}} u(\tau), \ldots,{ }^{c} D^{\beta_{n}} u(\tau)\right) d \tau\right) d s \\
& +\int_{t_{1}}^{t_{2}} \frac{\left(t_{2}-s\right)^{-\beta_{i}}}{\Gamma\left(1-\beta_{i}\right)}\left(\int_{0}^{s} \frac{(s-\tau)^{\alpha-2}}{\Gamma(\alpha-1)}\right. \\
& \times f\left(\tau, u(\tau),(\phi u)(\tau),(\psi u)(\tau),{ }^{c} D^{\beta_{1}} u(\tau),{ }^{c} D^{\beta_{2}} u(\tau), \ldots,{ }^{c} D^{\beta_{n}} u(\tau)\right) d \tau \\
& -\frac{b}{1+b} \int_{0}^{1} \frac{(1-\tau)^{\alpha-2}}{\Gamma(\alpha-1)} \\
& \left.\times f\left(\tau, u(\tau),(\phi u)(\tau),(\psi u)(\tau),{ }^{c} D^{\beta_{1}} u(\tau),{ }^{c} D^{\beta_{2}} u(\tau), \ldots,{ }^{c} D^{\beta_{n}} u(\tau)\right) d \tau\right) d s \mid \\
& \leq \int_{0}^{t_{1}} \frac{\left(t_{1}-s\right)^{-\beta_{i}}-\left(t_{2}-s\right)^{-\beta_{i}}}{\Gamma\left(1-\beta_{i}\right)} \\
& \times\left(\int_{0}^{s} \frac{(s-\tau)^{\alpha-2}}{\Gamma(\alpha-1)} \mid f(\tau, u(\tau),(\phi u)(\tau),(\psi u)(\tau),\right. \\
& \left.\left.{ }^{c} D^{\beta_{1}} u(\tau),{ }^{c} D^{\beta_{2}} u(\tau), \ldots,{ }^{c} D^{\beta_{n}} u(\tau)\right) \mid d \tau\right) d s \\
& +\frac{|b|}{|1+b|} \int_{0}^{t_{1}} \frac{\left(t_{1}-s\right)^{-\beta_{i}}-\left(t_{2}-s\right)^{-\beta_{i}}}{\Gamma\left(1-\beta_{i}\right)} \\
& \times\left(\int_{0}^{1} \frac{(1-\tau)^{\alpha-2}}{\Gamma(\alpha-1)} \mid f(\tau, u(\tau),(\phi u)(\tau),(\psi u)(\tau),\right. \\
& \left.\left.{ }^{c} D^{\beta_{1}} u(\tau),{ }^{c} D^{\beta_{2}} u(\tau), \ldots,{ }^{c} D^{\beta_{n}} u(\tau)\right) \mid d \tau\right) d s \\
& +\int_{t_{1}}^{t_{2}} \frac{\left(t_{2}-s\right)^{-\beta_{i}}}{\Gamma\left(1-\beta_{i}\right)}\left(\int_{0}^{s} \frac{(s-\tau)^{\alpha-2}}{\Gamma(\alpha-1)}\right. \\
& \left.\times\left|f\left(\tau, u(\tau),(\phi u)(\tau),(\psi u)(\tau),{ }^{c} D^{\beta_{1}} u(\tau),{ }^{c} D^{\beta_{2}} u(\tau), \ldots,{ }^{c} D^{\beta_{n}} u(\tau)\right)\right| d \tau\right) d s \\
& +\frac{|b|}{|1+b|} \int_{t_{1}}^{t_{2}} \frac{\left(t_{2}-s\right)^{-\beta_{i}}}{\Gamma\left(1-\beta_{i}\right)}\left(\int_{0}^{1} \frac{(1-\tau)^{\alpha-2}}{\Gamma(\alpha-1)}\right.
\end{aligned}
$$




$$
\begin{aligned}
& \left.\times\left|f\left(\tau, u(\tau),(\phi u)(\tau),(\psi u)(\tau),{ }^{c} D^{\beta_{1}} u(\tau),{ }^{c} D^{\beta_{2}} u(\tau), \ldots,{ }^{c} D^{\beta_{n}} u(\tau)\right)\right| d \tau\right) d s \\
\leq & \frac{N}{\Gamma(\alpha)} \int_{0}^{t_{1}} \frac{\left(t_{1}-s\right)^{-\beta_{i}}-\left(t_{2}-s\right)^{-\beta_{i}}}{\Gamma\left(1-\beta_{i}\right)} s^{\alpha-1} d s \\
& +\frac{N|b|}{|1+b| \Gamma(\alpha)} \int_{0}^{t_{1}} \frac{\left(t_{1}-s\right)^{-\beta_{i}}-\left(t_{2}-s\right)^{-\beta_{i}}}{\Gamma\left(1-\beta_{i}\right)} d s \\
& +\frac{N}{\Gamma(\alpha)} \int_{t_{1}}^{t_{2}} \frac{\left(t_{2}-s\right)^{-\beta_{i}}}{\Gamma\left(1-\beta_{i}\right)} s^{\alpha-1} d s+\frac{N|b|}{|1+b| \Gamma(\alpha)} \int_{t_{1}}^{t_{2}} \frac{\left(t_{2}-s\right)^{-\beta_{i}}}{\Gamma\left(1-\beta_{i}\right)} d s \\
\leq & \frac{(1+2|b|) N}{|1+b| \Gamma(\alpha)} \int_{0}^{t_{1}} \frac{\left(t_{1}-s\right)^{-\beta_{i}}-\left(t_{2}-s\right)^{-\beta_{i}}}{\Gamma\left(1-\beta_{i}\right)} d s \\
& +\frac{(1+2|b|) N}{|1+b| \Gamma(\alpha)} \int_{t_{1}}^{t_{2}} \frac{\left(t_{2}-s\right)^{-\beta_{i}}}{\Gamma\left(1-\beta_{i}\right)} d s \\
\leq & \frac{(1+2|b|) N}{|1+b| \Gamma(\alpha) \Gamma\left(2-\beta_{i}\right)}\left[\left(t_{2}^{1-\beta_{i}}-t_{1}^{1-\beta_{i}}\right)+2\left(t_{2}-t_{1}\right)^{1-\beta_{i}}\right] .
\end{aligned}
$$

Hence,

$$
\begin{aligned}
& \left\|F u\left(t_{2}\right)-F u\left(t_{1}\right)\right\| \\
& \leq \frac{N}{\Gamma(\alpha+1)}\left(t_{2}^{\alpha}-t_{1}^{\alpha}\right)+\frac{N|b|}{|1+b| \Gamma(\alpha)}\left(t_{2}-t_{1}\right) \\
& \quad+\sum_{i=1}^{n} \frac{(1+2|b|) N}{|1+b| \Gamma(\alpha) \Gamma\left(2-\beta_{i}\right)}\left[\left(t_{2}^{1-\beta_{i}}-t_{1}^{1-\beta_{i}}\right)+2\left(t_{2}-t_{1}\right)^{1-\beta_{i}}\right]
\end{aligned}
$$

which implies that $\left\|F u\left(t_{2}\right)-F u\left(t_{1}\right)\right\| \rightarrow 0$ as $t_{1} \rightarrow t_{2}$. Thus, $F$ is equi-continuous and uniformly bounded. By using the Arzela-Ascoli theorem, one can get that $F$ is completely continuous. Now, by using Theorem $2.5, F$ has a fixed point in $B_{r}$. Therefore, problem (1) has a solution.

Corollary 2.7 Let $f:[0,1] \times \mathbb{R}^{n+3} \rightarrow \mathbb{R}$ be a continuous function such that there exists a constant $l \in(0, \alpha-1)$ and a real-valued function $m(t) \in L^{\frac{1}{l}}([0,1],(0, \infty))$ such that $\left|f\left(t, x, y, w, u_{1}, u_{2}, \ldots, u_{n}\right)\right| \leq m(t)$ for all $t \in[0,1]$ and $x, y, w, u_{1}, u_{2}, \ldots, u_{n} \in \mathbb{R}$. Then problem (1) has a solution.

Finally, we should emphasize the importance of anti-periodic conditions, which appear in the case that $a=1$ and $b=1$ in the problem. For more details about this note, one can review the papers [24] and [25].

\subsection{Examples}

Example 2.1 Consider the boundary value problem

$$
\left\{\begin{aligned}
&{ }^{c} D^{\frac{7}{4}} u(t)= \frac{e^{-\pi t}}{24 \sqrt{\pi}+e^{-\pi t}}\left[\frac{\sin t+e^{t}}{1+t^{3}}+\frac{|u(t)|}{1+|u(t)|}+\frac{e^{-\pi t} \cos \pi t}{1+t^{2}}\left(1+\frac{\left|(\phi u)(t)++^{c} D^{\frac{1}{2}} u(t)\right|}{1+\left|(\phi u)(t)++^{c} D^{\frac{1}{2}} u(t)\right|}\right)\right. \\
&\left.+\frac{1+\sin ^{2} \pi t}{2\left(t^{\frac{3}{2}}+4\right)}\left((\psi u)(t)+\frac{\left|{ }^{c} D^{\frac{3}{4}} u(t)\right|}{1+\left|{ }^{c} D^{\frac{3}{4}} u(t)\right|}\right)\right], \\
& u(0)+u(1)=0, \quad u^{\prime}(0)+u^{\prime}(1)=0,
\end{aligned}\right.
$$


where $(\phi u)(t)=\int_{0}^{t} \frac{e^{-(s-t)}}{8} u(s) d s$ and $(\psi u)(t)=\int_{0}^{t} \frac{e^{-(s-t) / 2}}{8} u(s) d s$ with $\gamma_{0}=\frac{e-1}{8}$ and $\lambda_{0}=\frac{\sqrt{e}-1}{4}$. Then, we have

$$
\begin{aligned}
& \mid f\left(t, u(t),(\phi u)(t),(\psi u)(t),{ }^{c} D^{\frac{1}{2}} u(t),{ }^{c} D^{\frac{3}{4}} u(t)\right) \\
& \quad-f\left(t, v(t),(\phi v)(t),(\psi v)(t),{ }^{c} D^{\frac{1}{2}} v(t),{ }^{c} D^{\frac{3}{4}} v(t)\right) \mid \\
& \leq \frac{1}{24 \sqrt{\pi}}(|u(t)-v(t)|+|(\phi u)(t)-(\phi v)(t)| \\
&+|(\psi u)(t)-(\psi v)(t)|+\left|{ }^{c} D^{\frac{1}{2}} u(t)-{ }^{c} D^{\frac{1}{2}} v(t)\right| \\
&\left.+\left|{ }^{c} D^{\frac{3}{4}} u(t)-{ }^{c} D^{\frac{3}{4}} v(t)\right|\right) .
\end{aligned}
$$

Put $\mu(t)=\frac{1}{24 \sqrt{\pi}} \in L^{4}([0,1],(0, \infty)), \kappa=\frac{1}{4}$ and $\mu^{*}=\left(\int_{0}^{1}\left(\frac{1}{24 \sqrt{\pi}}\right)^{4} d s\right)^{\frac{1}{4}}=\frac{1}{24 \sqrt{\pi}}$. Since $\Gamma\left(\frac{3}{2}\right)=$ $\frac{\sqrt{\pi}}{2}, \Gamma\left(\frac{3}{4}\right) \approx 1 / 2,254, \Gamma\left(\frac{5}{4}\right) \approx 0 / 9,064$ and $\Gamma\left(\frac{7}{4}\right) \approx 0 / 9,191$, we have

$$
\begin{aligned}
&(1+\left.\gamma_{0}+\lambda_{0}\right)\left[\frac{3 \mu^{*}}{2 \Gamma(\alpha)}\left(\frac{1-\kappa}{\alpha-\kappa}\right)^{1-\kappa}+\frac{\mu^{*}}{4 \Gamma(\alpha-1)}\left(\frac{1-\kappa}{\alpha-\kappa-1}\right)^{1-\kappa}\right. \\
&+\frac{\Gamma(\alpha-\kappa) \mu^{*}}{\Gamma(\alpha-1)}\left(\frac{1-\kappa}{\alpha-\kappa-1}\right)^{1-\kappa}\left(\frac{1}{\Gamma\left(\alpha-\beta_{1}-\kappa+1\right)}+\frac{1}{\Gamma\left(\alpha-\beta_{2}-\kappa+1\right)}\right) \\
&\left.+\frac{\mu^{*}}{2 \Gamma(\alpha-1)}\left(\frac{1-\kappa}{\alpha-\kappa-1}\right)^{1-\kappa}\left(\frac{1}{\Gamma\left(2-\beta_{1}\right)}+\frac{1}{\Gamma\left(2-\beta_{2}\right)}\right)\right] \\
&=\left(1+\frac{e-1}{8}+\frac{\sqrt{e}-1}{4}\right)\left[\frac{1}{16 \sqrt{\pi} \Gamma\left(\frac{7}{4}\right)}\left(\frac{1}{2}\right)^{\frac{3}{4}}+\frac{1}{96 \sqrt{\pi} \Gamma\left(\frac{3}{4}\right)}\left(\frac{3}{2}\right)^{\frac{3}{4}}\right. \\
&\left.+\frac{\Gamma\left(\frac{3}{2}\right)}{24 \sqrt{\pi} \Gamma\left(\frac{3}{4}\right)}\left(\frac{3}{2}\right)^{\frac{3}{4}}\left(\frac{1}{\Gamma(2)}+\frac{1}{\Gamma\left(\frac{7}{4}\right)}\right)+\frac{1}{48 \sqrt{\pi} \Gamma\left(\frac{3}{4}\right)}\left(\frac{3}{2}\right)^{\frac{3}{4}}\left(\frac{1}{\Gamma\left(\frac{3}{2}\right)}+\frac{1}{\Gamma\left(\frac{5}{4}\right)}\right)\right] \\
& \approx 0 / 1,466<1 .
\end{aligned}
$$

Thus, by using Theorem 2.3, the boundary value problem (2) has a unique solution.

Example 2.2 Consider the boundary value problem

$$
\left\{\begin{aligned}
&{ }^{c} D^{\alpha} u(t)= \frac{\lambda e^{-\pi t}}{\sqrt{1+t^{2}}}+\frac{\cos \pi t}{\sqrt{\pi+|u(t)|+\left.\right|^{c} D^{\frac{1}{2}} u(t) \mid}}(u(t))^{\sigma_{1}}+\frac{e^{-\pi t}\left(1+\sin ^{2} u(t)\right)}{(t+6)^{3}}((\phi u)(t))^{\sigma_{2}} \\
&+\frac{t u(t)}{\left(4+t^{2}\right)(1+|u(t)|)}((\psi u)(t))^{\sigma_{3}} \\
&+\frac{(1+\alpha)\left(t-\frac{1}{2}\right)^{2}}{\Gamma(\alpha)\left(1+\left|u(t)+{ }^{c} D^{\frac{3}{2}} u(t)\right|\right)} \sum_{k=1}^{4}\left(\frac{\sin k \pi t}{2^{k}}\right)\left({ }^{c} D^{\beta_{k}} u(t)\right)^{\delta_{k}}, \\
& u(0)+\frac{1}{2} u(1)=0, \quad u^{\prime}(0)+\frac{2}{3} u^{\prime}(1)=0
\end{aligned}\right.
$$

where $\alpha=\frac{9}{5}, \beta_{1}=\frac{1}{2}, \beta_{2}=\frac{4}{5}, \beta_{3}=\frac{2}{3}, \beta_{4}=\frac{1}{7}, \lambda \in[0, \infty),(\phi u)(t)=\int_{0}^{t} \frac{s^{2} e^{-(s-t) / 2}}{s^{3}+4} u(s) d s$ and $(\psi u)(t)=\int_{0}^{t} \frac{(t-s)^{5}}{\sqrt{1+s^{2}}} u(s) d s$. Then, we have

$$
\begin{aligned}
& \left|f\left(t, u(t),(\phi u)(t),(\psi u)(t),{ }^{c} D^{\beta_{1}} u(t),{ }^{c} D^{\beta_{2}} u(t),{ }^{c} D^{\beta_{3}} u(t),{ }^{c} D^{\beta_{4}} u(t)\right)\right| \\
& \quad \leq m(t)+\frac{1}{\sqrt{\pi}}|u(t)|^{\sigma_{1}}+\frac{1}{108}|(\phi u)(t)|^{\sigma_{2}}+\frac{1}{4}|(\psi u)(t)|^{\sigma_{3}}+\sum_{k=1}^{4} \frac{(1+\alpha)}{\Gamma(\alpha) 2^{k+2}}\left|{ }^{c} D^{\beta_{k}} u(t)\right|^{\delta_{k}},
\end{aligned}
$$


where $m(t)=\frac{\lambda e^{-\pi t}}{\sqrt{1+t^{2}}}$ for all $t \in[0,1]$. If $0<\sigma_{j}<1,0<\delta_{i}<1$ for $j=1,2,3$ and $i=1,2,3,4$, then assumption $(*)$ holds. If $\lambda=0$ and $\sigma_{j}, \delta_{i}>1$ for $j=1,2,3$ and $i=1,2,3,4$, then the second condition of Theorem 2.6 holds. Thus, by using Theorem 2.6 , the boundary value problem (3) has a solution.

\section{Conclusions}

Fractional nonlinear differential equations, fractional integro-differential equations and their applications represent a topic of high interest in the area of fractional calculus and its applications in various fields of science and engineering [7]. In this article, based on the Schauder's fixed point theorem, we have proved some existence results for the multi-term nonlinear fractional integro-differential equation (1).

\section{Competing interests}

The authors declare that they have no competing interests.

\section{Authors' contributions}

All authors have equal contributions. All authors read and approved the final manuscript.

\section{Author details}

'Department of Chemical and Materials Engineering, Faculty of Engineering, King Abdulaziz University, P.O. Box 80204 Jeddah, 21589, Saudi Arabia. ${ }^{2}$ Department of Mathematics, Cankaya University, Ogretmenler Cad. 14, Balgat, Ankara, 06530, Turkey. ${ }^{3}$ Institute of Space Sciences, Magurele, Bucharest, Romania. ${ }^{4}$ Department of Mathematics, Azarbaijan Shahid Madani University, Azarshahr, Tabriz, Iran.

\section{Acknowledgements}

Research of the second and the third authors was supported by Azarbaijan Shahid Madani University. Also, the authors express their gratitude to the referees for their helpful suggestions, which improved the final version of this paper.

Received: 21 March 2013 Accepted: 28 August 2013 Published: 13 Dec 2013

\section{References}

1. Hilfer, R: Applications of Fractional Calculus in Physics. World Scientific, Singapore (2000)

2. Kilbas, AA, Srivastava, HM, Trujillo, JJ: Theory and Applications of Fractional Differential Equations. North-Holland Mathematics Studied, vol. 204. Elsevier, Amsterdam (2006)

3. Podlubny, I: Fractional Differential Equations. Mathematics in Science and Engineering. Academic Press, San Diego (1999)

4. Ahmad, B: Existence of solutions for fractional differential equations of order $q \in(2,3)$ with anti-periodic boundary conditions. J. Appl. Math. Comput. 34, 385-391 (2009)

5. Baleanu, D, Agarwal, RP, Mohammadi, H, Rezapour, S: Some existence results for a nonlinear fractional differential equation on partially ordered Banach spaces. Bound. Value Probl. 2013, 112 (2013)

6. Baleanu, D, Mohammadi, H, Rezapour, S: Positive solutions of a boundary value problem for nonlinear fractional differential equations. Abstr. Appl. Anal. 2012, Article ID 837437 (2012)

7. Baleanu, D, Mohammadi, H, Rezapour, S: Some existence results on nonlinear fractional differential equations. Philos. Trans. R. Soc. Lond. A 371, 20120144 (2013)

8. Baleanu, D, Mohammadi, H, Rezapour, S: On a nonlinear fractional differential equation on partially ordered metric spaces. Adv. Differ. Equ. 2013, 83 (2013)

9. Chang, YK, Nieto, JJ: Some new existence results for fractional differential inclusions with boundary conditions. Math. Comput. Model. 49, 605-609 (2009)

10. Chen, A, Chen, Y: Existence of solutions to anti-periodic boundary value problem for nonlinear fractional differential equations. Differ. Equ. Dyn. Syst. 19(3), 237-252 (2011)

11. Davi, JV, Lakshmikantham, V: Nonsmooth analysis and fractional differential equations. Nonlinear Anal. 69, 2677-2682 (2008)

12. Lakshmikantham, V, Vatsala, AS: Basic theory of fractional differential equations. Nonlinear Anal. 69, 2677-2682 (2008)

13. Li, CF, Luo, XN, Zhou, Y: Existence of positive solutions of the boundary value problem for nonlinear fractional differential equations. Comput. Math. Appl. 59, 1363-1375 (2010)

14. Sun, S, Li, Q, Li, Y: Existence and uniqueness of solutions for a coupled system of multi-term nonlinear fractional differential equations. Comput. Math. Appl. (2012). doi:10.1016/j.camwa.2012.01.065

15. Jaradat, O, Al-Omari, A, Momani, SM: Existence of the mild solution for fractional semilinear initial value problems. Nonlinear Anal. 69, 3153-3159 (2008)

16. Momani, SM, El-Khazali, R: On the existence of extremal solutions of fractional integro-differential equations. J. Fract. Calc. 18, 87-92 (2000)

17. Momani, SM: Some existence theorems on fractional integro-differential equations. Abhath Al-Yarmouk J. 10, 435-444 (2001)

18. Momani, SM, Hadid, SB: On the inequalities of integro-differential fractional equations. Int. J. Appl. Math. 12(1), 29-37 (2003) 
19. Momani, SM, Hadid, SB: Some comparison results for integro-fractional differential inequalities. J. Fract. Calc. 24, 379-387 (2003)

20. Xinwei, S, Landong, L: Existence of solution for boundary value problem of nonlinear fractional differential equation. Appl. Math. J. Chin. Univ. Ser. B 22(3), 291-298 (2007)

21. Su, X, Zhang, S: Solutions to boundary value problems for nonlinear differential equations of fractional order. Electron. J. Differ. Equ. 2009, 26 (2009)

22. Ahmad, B, Sivasundaram, S: On four-point nonlocal boundary value problems of nonlinear integro-differential equations of fractional order. Appl. Math. Comput. 217, 480-487 (2010)

23. Agarwal, RP, O'Regan, D, Sahu, DR: Fixed Point Theory for Lipschitzian-Type Mappings with Applications. Springer, Berlin (2009)

24. Ahmad, B: Nonlinear fractional differential equations with anti-periodic type fractional boundary conditions. Differ. Equ. Dyn. Syst. (2012). doi:10.1007/s12591-012-0154-2

25. Ahmad, B, Nieto, JJ: Anti-periodic fractional boundary value problems with nonlinear term depending on lower order. Fract. Calc. Appl. Anal. 15, 451-462 (2012)

10.1186/1687-1847-2013-368

Cite this article as: Baleanu et al.: Existence and uniqueness of solutions for multi-term nonlinear fractional integro-differential equations. Advances in Difference Equations 2013, 2013:368

\section{Submit your manuscript to a SpringerOpen ${ }^{\circ}$ journal and benefit from:}

- Convenient online submission

- Rigorous peer review

- Immediate publication on acceptance

- Open access: articles freely available online

- High visibility within the field

- Retaining the copyright to your article 